\title{
Nivel de adecuación del territorio para el desarrollo de especies agrícolas y forestales en el Parque Nacional Nevado de Toluca
}

\section{Territorial suitability level for the development of farming and forest species in the Nevado de Toluca National Park}

\author{
Héctor Hugo Regil-García \\ Sergio Franco-MaAss*
}

\begin{abstract}
The Nevado de Toluca National Park is an important reserve of natural resources in the State of Mexico. The recovering of forest ecosystems was established as an objective to determine appropriateness level for the development of native forest species and crops in the area. We present a methodology based on the use of Geographic Information Systems (GIS) to evaluate some of the needs (i.e. climatic, physiographic, edaphic) of those species and to generate suitability criteria maps. This analysis provided an optimal occupation of the soil for four forest and two farming species.
\end{abstract}

Keywords: territorial suitability level, geographic information systems (GIS), ecosystem recovery.

\section{Resumen}

El Parque Nacional Nevado de Toluca es una importante reserva de recursos naturales del Estado de México. En la recuperación de los ecosistemas forestales se planteó como objetivo determinar el grado de adecuación para el desarrollo de especies forestales nativas y cultivos en la zona. En este trabajo se presenta una metodología basada en la aplicación de sistemas de información geográfica (SIG) para evaluar algunos requerimientos de las especies (climáticos, fisiográficos y edáficos) y generar mapas criterios de adecuación. El análisis permitió obtener la ocupación óptima del suelo de cuatro especies forestales y dos especies agrícolas.

Palabras clave: grado de adecuación del territorio, sistemas de información geográfica (SIG), recuperación de ecosistemas.

* Universidad Autónoma del Estado de México, México. Correos e: regil_hh@yahoo.com.mx; sfrancom@uaemex.mx. 


\section{Introducción}

Por medio de decreto, en 1936 se creó el Parque Nacional Nevado de Toluca (PNNT) con la finalidad de proteger los recursos naturales en torno al volcán Nevado de Toluca. A pesar de que se considera una de las principales zonas de reserva de recursos naturales del Estado de México y un espacio natural representativo de la biodiversidad de las montañas templadas en México, en la actualidad presenta importantes signos de deterioro. De acuerdo con Franco et al. (2006a), hacia el año 2000, poco más de 15\% de la superficie total del parque se encontraba cubierta por agricultura y cerca de $11.5 \%$ estaba ocupada por bosques seriamente deteriorados. Ante esta situación se presentan, año con año, esfuerzos gubernamentales y de la población para revertir estos procesos de deterioro mediante esquemas de reforestación. Dichos esfuerzos, sin embargo, no han mostrado ser efectivos y no toman en consideración la reintroducción de especies nativas con miras a la recuperación de los ecosistemas naturales.

\section{Figura I}

\section{Ubicación del Parque Nacional Nevado de Toluca}

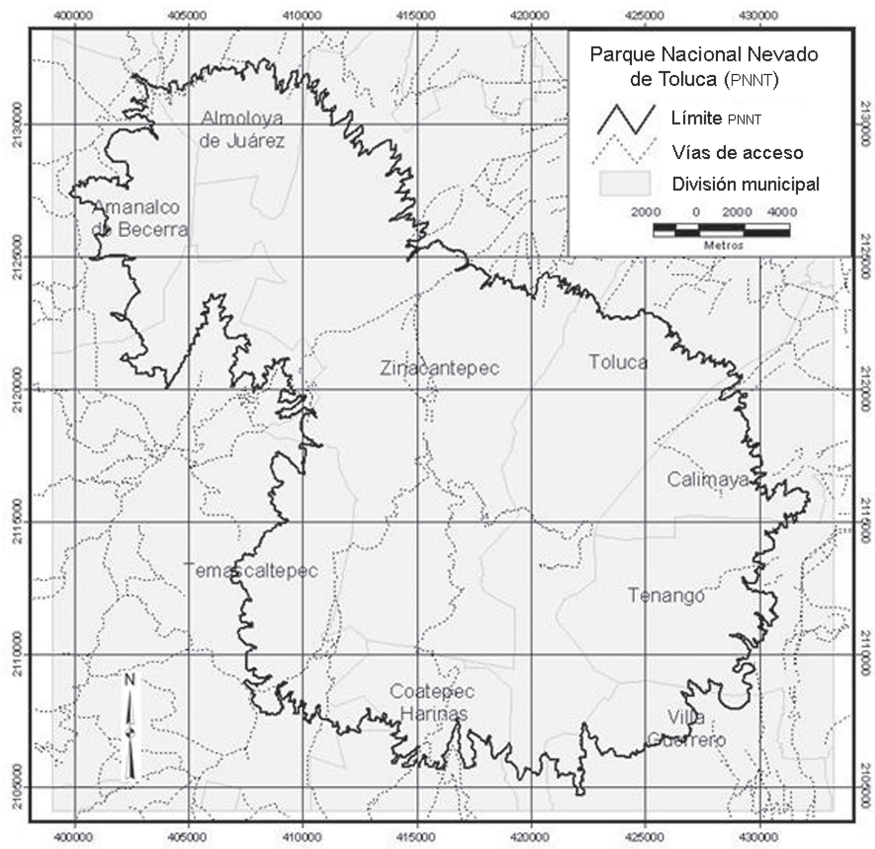

Fuente: Elaboración propia. 
Por su extensión, el PNNT es el principal parque nacional del Estado de México: representa 52\% del área total de los 10 parques nacionales ubicados en la entidad (GEM, 1999). Se localiza en el Sistema Volcánico Transversal y abarca parte de los municipios de Zinacantepec, Toluca, Calimaya, Tenango del Valle, Villa Guerrero, Coatepec Harinas, Almoloya de Juárez, Temascaltepec y Amanalco de Becerra (figura I). El área natural protegida se localiza entre 2'102,398 y 2'134,700 metros norte y 399,833 y 432,253 metros este en coordenadas UTM.

El parque cuenta con un patrimonio natural muy importante y constituye un proveedor neto de servicios ambientales, como la regulación del ciclo hidrológico, la conservación de suelos, la captura de carbono, la aportación de oxígeno a la atmósfera y soporte de actividades turísticas, recreativas y de investigación.

A pesar de su carácter de área natural protegida (ANP), este parque se encuentra sujeto a una intensa intervención humana que se refleja en las actividades agrícolas en zonas de montaña con fuertes pendientes y poca aptitud productiva, lo que ha afectado tanto la configuración natural del parque como la economía local, debido a la baja productividad de sus cultivos.

El Parque Nacional Nevado de Toluca se ha estudiado desde varias vertientes: su fauna, su vegetación, el deterioro de la biodiversidad forestal, las descripciones físicas, el uso de suelo y la elaboración de planes de manejo. Sin embargo, se desconoce el estado de adecuación del territorio para el desarrollo de las especies vegetales nativas y para el crecimiento de los principales cultivos en la región. No obstante su importancia en el ordenamiento ecológico, se carece de una metodología que permita obtener, de manera cuantitativa, el nivel de adecuación del territorio, entendido éste como el grado de idoneidad o cabida que presenta dicho territorio para una cierta actividad, teniendo en cuenta la medida en la que el medio cubre los requisitos locacionales de la actividad y los efectos de tal actividad sobre el medio.

Con base en lo anterior se planteó, como objetivo central de esta investigación, determinar el grado de adecuación del Parque Nacional Nevado de Toluca para el desarrollo de las principales especies forestales nativas (Pinus harwegii, P. montezumae, $P$ ayacahuite, Abies religiosa, Alnus acuminata y Quercus laurina). Asimismo, se buscó analizar el nivel de adecuación para el desarrollo de los principales cultivos que se practican en la zona: papa (Solanum tuberosum), avena (Avena sativa), maíz (Zea mays) y haba (Vicia faba). El cumplimiento de estos objetivos implicó el 
desarrollo de una metodología basada en la aplicación de los sistemas de información geográfica (SIG) y en las técnicas de evaluación multicriterio (EMC). En primer lugar fue necesario caracterizar los principales requerimientos de las diversas especies forestales y agrícolas (climáticos, fisiográficos y edáficos). Con base en dicha información, mediante el uso de un programa de SIG, fue posible generar un conjunto de mapas criterios de adecuación (factores y restricciones) que, analizados mediante técnicas EMC, permitieron obtener el grado de adecuación del territorio para el desarrollo de cada una de dichas especies. Después se hizo un análisis comparativo de los niveles de adecuación por especie que permitió obtener la ocupación óptima del suelo para las cuatro especies forestales más significativas y, finalmente, se analizaron las zonas agrícolas en relación con su nivel de adecuación para dicha actividad.

\section{La evaluación multicriterio}

La evaluación multicriterio (EMC) es un conjunto de técnicas que permiten evaluar diversas alternativas de elección a la luz de múltiples criterios y prioridades. Desde el punto de vista espacial, estas alternativas son unidades de observación o porciones del territorio que se evalúan con base en sus características geográficas (Barredo, 1996).

La aplicación de la EMC permite asistir en los procesos de ordenamiento territorial mediante la obtención del nivel de adecuación del territorio para mantener los ecosistemas naturales y para desarrollar las actividades productivas. Surgió como respuesta al paradigma decisional de la investigación operativa, el cual planteaba como principio "la búsqueda de una decisión óptima maximizando una función económica" (Barba-Romero y Pomerol, 1997: 17).

En la EMC los problemas pueden requerir el planteamiento de un solo objetivo o también es posible que sean de carácter multiobjetivo. Por ello la definición de un conjunto de elección dependerá en buena medida del modelo de datos utilizado. En el ambiente vectorial se refiere a un conjunto de polígonos que deben ser evaluados. En el ambiente raster, por su parte, cada celda de la matriz se debe considerar como un elemento independiente.

Por otro lado, es importante establecer un criterio como el componente básico de la EMC, definido como "todo aspecto medible de un juicio, mediante el cual se puede caracterizar una de las dimen- 
siones de las diversas alternativas de elección bajo consideración" (Voogd, 1983: 21). De acuerdo con Eastman et al. (1993), un criterio es una cierta base que se puede medir y evaluar.

En un SIG las unidades de observación (expresadas como celdas o polígonos) cuentan con una serie de atributos cualitativos o nominales (ocupación del suelo, unidades edáficas, etc.) o cuantitativos (altitud, precipitación, etc.). Estos atributos por sí solos no reflejan las preferencias del decisor. En la EMC se aplica un procedimiento para traducir dichos atributos en puntuaciones $o$ valores de preferencia (criterios) (Franco, 2003).

En los SIG existen dos tipos de criterios:

Factor. Es un criterio que aumenta o disminuye a partir del nivel de adecuación de una alternativa específica para la actividad considerada y que, en general, se mide en una escala de razón, es decir, una escala de unidades de medida con "origen conocido, indicado por el número 'cero'” (Voogd, 1983: 23).

Restricción. Es un criterio que sirve como límite a las alternativas consideradas y que en muchos casos se representa en una "escala binaria la cual expresa un orden parcial dado que únicamente representa información 'si'-'no'” (Voogd, 1983: 23). Así, las áreas que se van a excluir de la evaluación reciben un valor de cero y las zonas consideradas tienen un valor de uno.

Estos dos criterios se utilizaron en el presente estudio.

\section{Método general para determinar el nivel de adecuación del territorio}

El nivel de adecuación del territorio se concibe como "el grado de idoneidad o cabida que presenta el territorio para una actividad, teniendo en cuenta a la vez, la medida en que el medio cubre sus requisitos locacionales y los efectos de dicha actividad sobre el medio" (Gómez, 1992: 264). El concepto de nivel de adecuación del territorio se ha retomado en varios estudios de muy diversa índole. Sánchez y Monte (1992) determinaron la capacidad de uso de suelo para equipamientos urbanos. Luque (2003) localizó los lugares óptimos para establecer actividades turísticas. Desde el punto de vista agroforestal destaca la aportación de Santé y Crecente (2005), quienes realizaron una aplicación de sIG para establecer sistemas agroforestales.

Como se mencionó, esta investigación se basa en la obtención del nivel de adecuación del territorio para desarrollar diversas especies forestales y agrícolas en el PNNT. Con la finalidad de 
cumplir con este objetivo fue necesario llevar a cabo una serie de procedimientos para obtener y analizar mapas digitales de clima, fisiografía y edafología mediante la aplicación de diversos programas de SIG. En la figura II se muestra el esquema general de la investigación.

Figura 2

Diseño de investigación

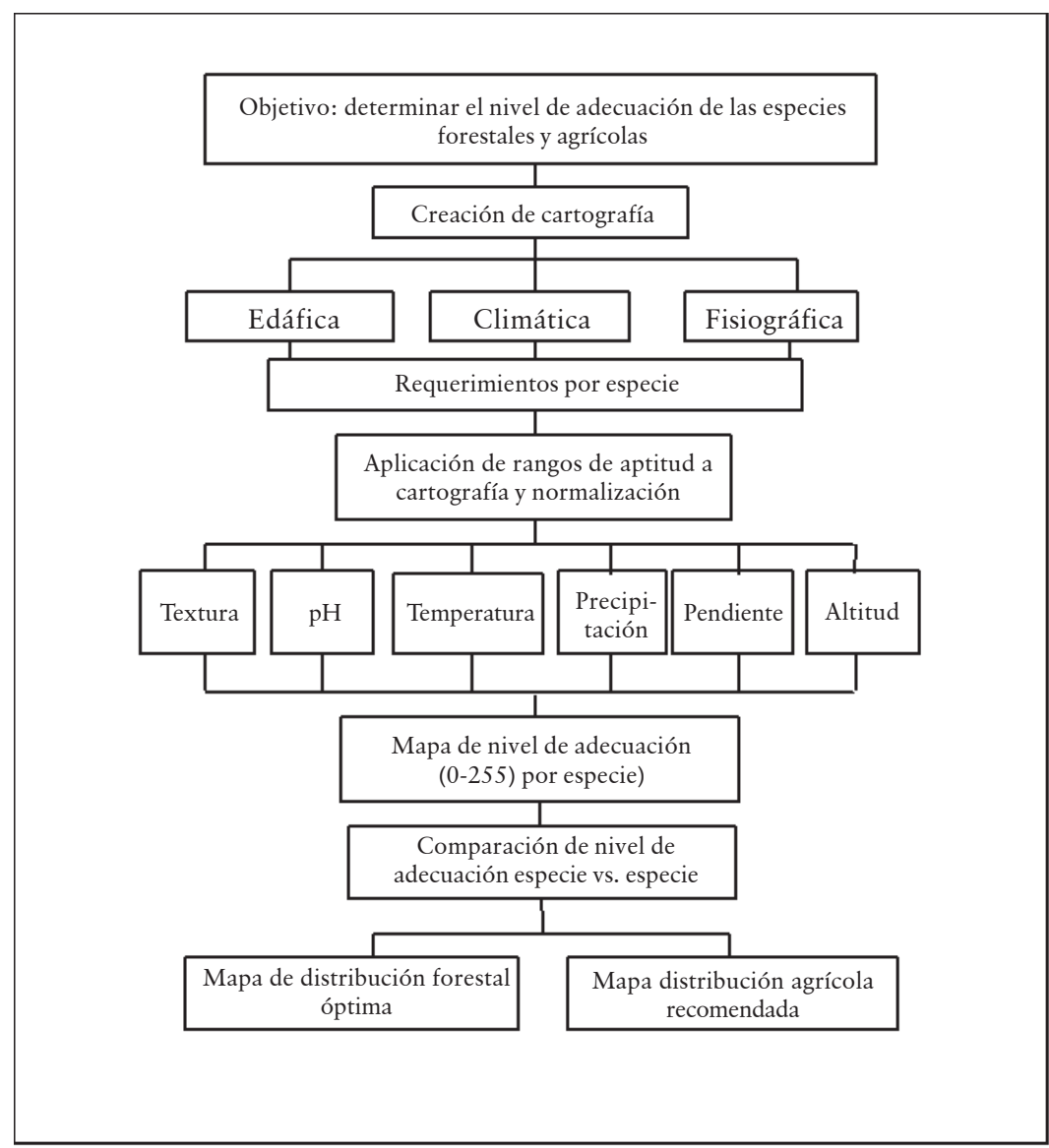

Fuente: Elaboración propia.

\subsection{Obtención de los requerimientos por especie}

La investigación documental permitió conocer las necesidades edáficas, climáticas y fisiográficas más importantes de cada una de las principales especies forestales y agrícolas reportadas para el Parque Nacional Nevado de Toluca. 


\subsubsection{Requerimientos edáficos}

Para obtener las tablas de adecuación edáfica se utilizaron las fichas técnicas para reforestación de la Conafor (2006), para el caso de las especies forestales, y para las especies agrícolas se usaron los datos de Aragón (2003), cuyos resultados se muestran en el cuadro 1.

\section{Cuadro 1}

\begin{tabular}{|c|c|c|}
\hline Especie & pH óptimo & Textura \\
\hline Pinus hartwegii & $6.1-6.9$ & Franco-arenosa \\
\hline Abies religiosa & $5.0-7.0$ & $\begin{array}{l}\text { Limo-arenosa, arcillo-arenosa, } \\
\text { arenosa }\end{array}$ \\
\hline Pinus montezumae & $5.0-7.0$ & $\begin{array}{l}\text { Franco-arenosa, arenosa, } \\
\text { areno-limosa }\end{array}$ \\
\hline Pinus ayacahuite & $5.0-8.0$ & $\begin{array}{l}\text { Arcillosa, franco-arcillosa, } \\
\text { franco-limosa, franco arenosa }\end{array}$ \\
\hline Alnus acuminata & $4.0-6.0$ & Arenosa o arcillosa \\
\hline Quercus laurina & $4.8-7.5$ & $\begin{array}{l}\text { Limosa, arcillosa, franca- } \\
\text { arcillosa-arenosa, franca- } \\
\text { arenosa }\end{array}$ \\
\hline Solanum tuberosum & $\begin{array}{l}5.5 \text { a } 6 \\
\text { hasta } 4.4 \\
\text { ácido }\end{array}$ & $\begin{array}{l}\text { Franca, franco-arcillo-limosa, } \\
\text { franco-arenosa }\end{array}$ \\
\hline Avena sativa & 5.5 a 7.5 & $\begin{array}{l}\text { Cualquier textura, de preferencia } \\
\text { arcillo-limosa, franco-arcillosa, } \\
\text { limosa }\end{array}$ \\
\hline Zea mays & 6 a 7 & $\begin{array}{l}\text { Franco-limosa, franca arcillo- } \\
\text { limosa, Franco-arcilloso }\end{array}$ \\
\hline Vicia faba & 5 a 7 & Franco, franco arenoso \\
\hline
\end{tabular}

\subsubsection{Requerimientos climáticos}

$\mathrm{Al}$ igual que para el caso de las necesidades edáficas, para obtener las tablas de adecuación climática se utilizaron las mismas fuentes que para el cuadro 1 . Los resultados se presentan en el cuadro 2.

Cabe mencionar que los campos marcados con un asterisco fueron requerimientos corroborados en campo para corregir límites superiores o inferiores de adecuación. 

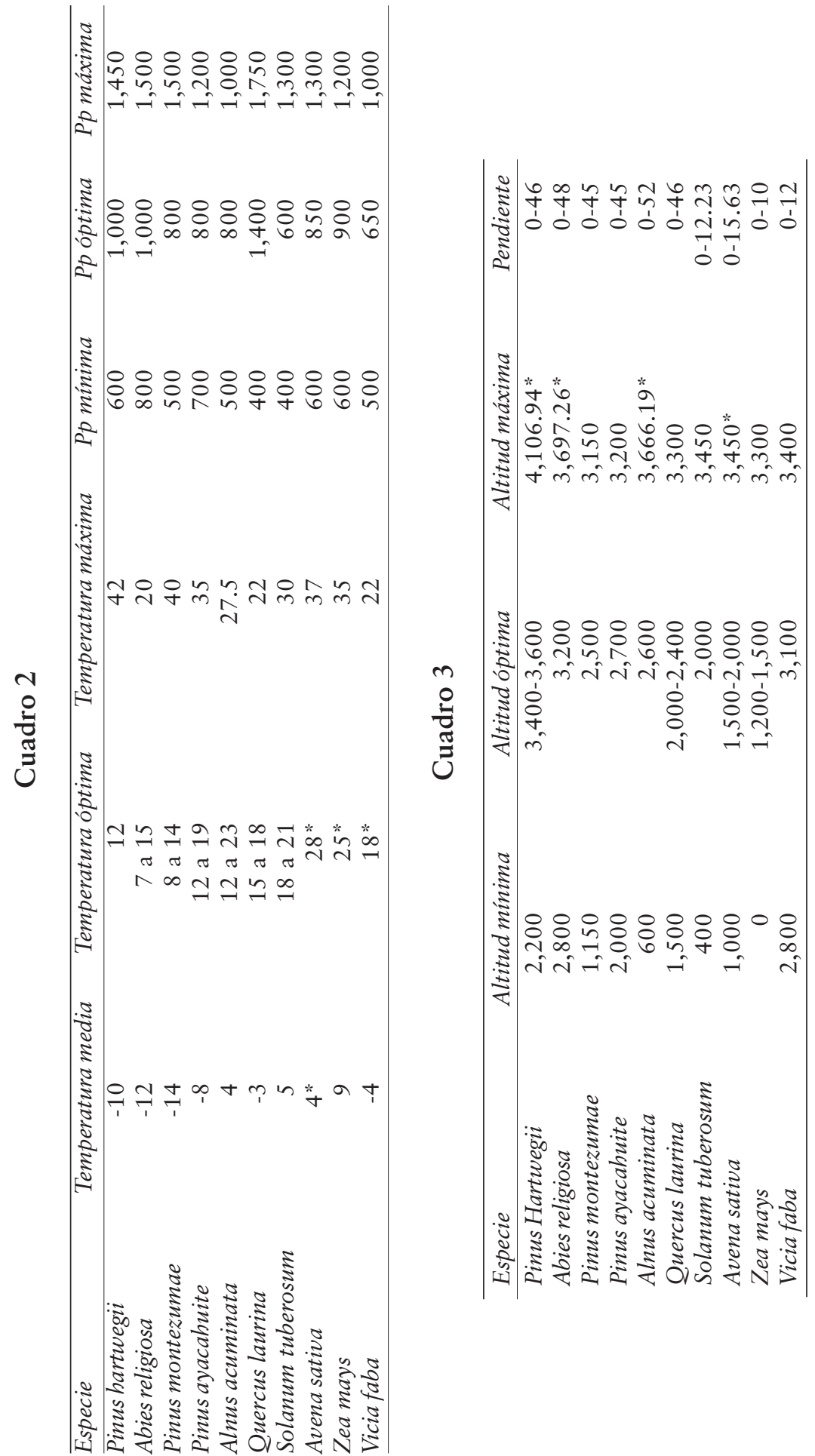


\subsubsection{Requerimientos fisiográficos}

$\mathrm{Al}$ igual que en los dos casos anteriores, para obtener las tablas de adecuación fisiográfica nos basamos en las mismas fuentes. Los resultados se muestran en el cuadro 3.

$\mathrm{Al}$ igual que en el caso anterior, los campos marcados con un asterisco, fueron requerimientos corroborados en campo para corregir límites superiores o inferiores de adecuación.

Para cada variable -salvo en el caso de los datos edáficos- se buscó obtener un dato de adecuación óptima y dos de adecuación mínima o marginal para cada especie, que permitiera en procesos posteriores no sólo determinar si un territorio es apto o no para acoger la especie vegetal, sino también en qué grado.

\subsection{Obtención de los mapas de criterios}

Como señala Malczewski (1999), los mapas de criterios en un SIG son todas aquellas coberturas que representan los criterios de evaluación, es decir, los atributos que se asocian con los objetivos. Para el caso de la evaluación del nivel de adecuación del parque nacional, se consideraron los requerimientos básicos edáficos, climáticos y fisiográficos, para obtener un conjunto de mapas de criterios. Es importante señalar que, para efectos de comparación y análisis, obtener los mapas de criterios requirió aplicar el procedimiento de normalización en la escala 0-255 recomendada por Eastman (1995). En esta escala, el grado máximo de adecuación tiene un valor de 255, y el grado nulo de adecuación es de cero.

\subsubsection{Cartografía edáfica}

De acuerdo con la información cartográfica disponible fue posible generar dos mapas de criterios edáficos: la cobertura del potencial de hidrógeno en el suelo $(\mathrm{pH})$ y la textura del suelo. Estos mapas se generaron a partir de la Carta de Unidades Edáficas, elaborada para el proyecto "Evaluación edafológico-climática para la introducción de frutales perennes y pastos retenedores de suelo en el Parque Nacional Nevado de Toluca" (Franco y Regil, 2005). Se obtuvieron dos mapas del área natural protegida: uno corresponde a dos categorías de textura (areno-franca y franca) y otro se refiere a 14 diferentes niveles de $\mathrm{pH}$ en función de las diferentes unidades edáficas. 


\subsubsection{Cartografía climática}

Los dos aspectos climáticos considerados fueron temperatura y precipitación. Dichas variables se obtuvieron a partir de los datos de 63 estaciones meteorológicas cercanas al área de estudio extraídas del programa ERIC 2 (IMTA, 1996). Los datos por estación meteorológica se revisaron y depuraron de acuerdo con la metodología reportada por García (1989). Una vez depurada la información por estación meteorológica fue posible aplicar procedimientos de interpolación para obtener los mapas de distribución de temperaturas medias anuales y de precipitación anual.

\subsubsection{Cartografía fisiográfica}

En cuanto a los aspectos fisiográficos, se elaboraron los mapas de altitud y pendientes, tomando como insumo principal las curvas de nivel digitales del INEGI, correspondientes a las cartas E14 A37, E14 A47 y A14 A48. Para ello fue necesario generar un modelo digital del terreno (MDT) mediante procedimientos de interpolación. El mapa de pendientes en grados es un producto derivado del MDT.

\subsection{Obtención de mapas de nivel de adecuación por especie}

Una vez que contamos con los mapas de criterios correspondientes a cada una de las especies en estudio, fue posible obtener el nivel de adecuación del territorio para cada una de ellas. El procedimiento básico utilizado fue el de la suma ponderada de los mapas de criterios descrito por Eastman (1995). En la figura III se muestra el procedimiento seguido para obtener un mapa de nivel de adecuación edáfico-climático-fisiográfico para cada especie forestal y agrícola. Como es posible observar, se partió de cinco mapas de aptitud por especie (precipitación, temperatura, $\mathrm{pH}$, pendiente y altitud). El paso siguiente consistió en ponderar el valor de cada variable en función de su importancia relativa y considerando que la suma de dichas ponderaciones fuera la unidad. Finalmente, la suma de dichos criterios previamente ponderados permitió derivar un mapa de adecuación global por especie, cuyos valores se distribuían entre 0 y 255. 


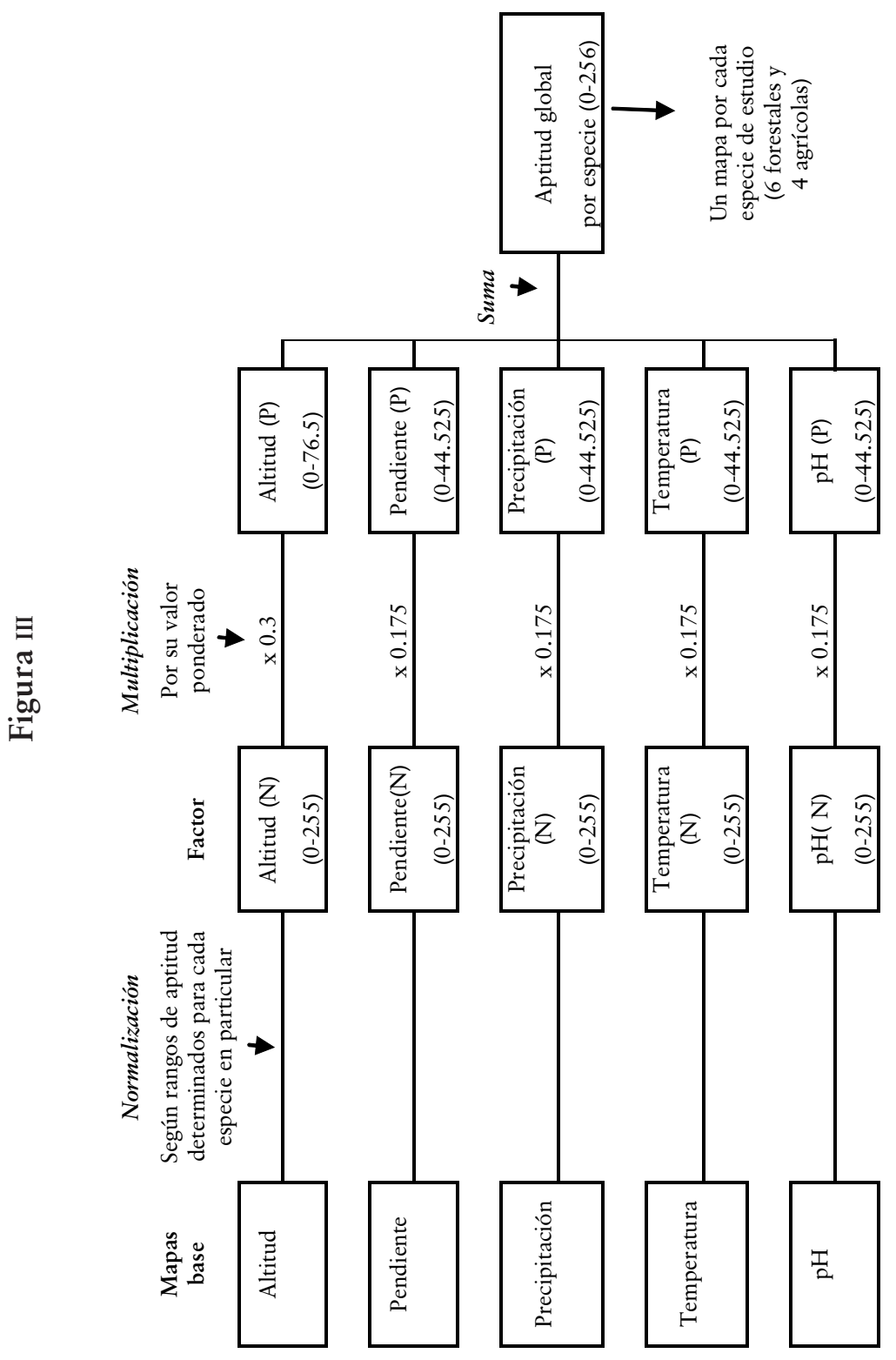




\subsection{Obtención de mapas de restricción}

Un aspecto a tomar en cuenta en cualquier análisis espacial es la presencia de zonas donde no es posible llevar a cabo la actividad propuesta. En estos casos el procedimiento básico consiste en considerar mapas de restricciones.

Para efectos de la investigación se incluyeron tres tipos de restricciones: textura, uso de suelo y altitud. En el primer caso se consideró que algunas especies no podían desarrollarse en suelos areno-francos y, por consiguiente, se debían excluir de los mapas de nivel de adecuación correspondientes a: Pinus ayacahuite, Quercus laurina, Zea mays, Solanum tuberosum y Vicia faba.

En el caso del uso de suelo se consideraron dos mapas de restricción. Para las especies forestales se tomó como limitante para su desarrollo las zonas altas del parque nacional con eriales y eriales asociados con pastizal alpino. Por otro lado, la restricción para especies agrícolas fue mayor e incluyó tanto la restricción de eriales y eriales con pastizal alpino, así como todas aquellas regiones con cobertura forestal, ya que no tenía sentido sugerir el establecimiento de cultivos en lugares que de manera natural tienen una cobertura arbórea.

En lo que respecta a la altitud se consideró como restricción toda altitud mayor al límite superior de la tabla de requerimientos de cada una de las especies seleccionadas.

Una vez obtenidas las coberturas de restricciones, éstas se multiplicaron a los mapas de nivel de adecuación por especie previamente obtenidos, de manera que el mapa resultante excluyera aquellas áreas determinadas donde no es posible desarrollar la especie.

\subsection{Obtención de la distribución forestal más adecuada}

En el entendido de que el área ocupada por el PNNT tiene una vocación eminentemente forestal, y a sabiendas de que ha sufrido diversos procesos antrópicos a lo largo del tiempo que le dan su actual configuración en cuanto a la ocupación del suelo, y después de obtener el nivel de adecuación de cada una de las especies, se procedió a comparar el nivel de adecuación de las principales especies forestales para determinar la configuración ideal del área protegida, tomando en cuenta las variables climáticas, fisiográficas y edáficas abordadas en este estudio. 
Para ello se llevó a cabo, en el ambiente SIG, un análisis de dominancia basado en la resta de coberturas. Las áreas con valores positivos son aquéllas donde la alternativa 1 domina sobre la alternativa 2 y, por consiguiente, el territorio es más adecuado para dicha especie.

Primero se jerarquizó a las cuatro especies según su importancia económica. De acuerdo con este enfoque, el orden jerárquico sería: 1) pino, 2) oyamel, 3) aile y 4) encino.

El proceso SIG elaborado en este caso sería una resta que, evidentemente, arrojaría valores positivos y negativos. Las áreas con valores positivos serán las zonas en que la especie 1 es más apta que la 2, y las áreas con valores negativos son las zonas en las que la especie 2 es más apta que la 1 (figura IV).

\section{Figura IV}

\begin{tabular}{|c|c|c|c|c|}
\hline \multicolumn{2}{|c|}{1} & \multirow[b]{2}{*}{ Resta } & \multicolumn{2}{|c|}{2} \\
\hline 233 & 235 & & 204 & 230 \\
\hline 233 & 237 & & 208 & 240 \\
\hline \multicolumn{3}{|c|}{ Aptitud Pinus hartwegii } & \multicolumn{2}{|c|}{ Aptitud Alnus acuminat } \\
\hline \multicolumn{2}{|c|}{4} & & \multicolumn{2}{|c|}{$\downarrow 3$} \\
\hline 1 & 1 & \multirow{2}{*}{ Reclasificación } & 29 & 5 \\
\hline 1 & 0 & & 3 & -3 \\
\hline \multicolumn{5}{|c|}{ Preferencia Pinus vs. Alnus } \\
\hline
\end{tabular}

Como se observa en la figura, los valores positivos fueron reclasificados para darle valor de 1 y los negativos con valor de 0 , pues sólo se quiere conocer en qué zonas la especie 1 es más apta que la dos. 


\subsection{Obtención de la distribución agrícola sugerida}

Una vez que se obtuvo la distribución forestal ideal, hay una consideración que no se podía pasar por alto: el hecho de que dentro del uso actual del PNNT existe un área agrícola considerable, la cual es la fuente de subsistencia de la población local, y por esto el hecho de proponer una reconversión a un uso forestal resulta inviable.

Tomando en cuenta esto, el proceso siguiente fue determinar en las zonas con un uso agrícola actual, la aptitud de los cultivos reportados, para así sugerir una distribución de acuerdo con su potencial climático, fisiográfico y edáfico.

Como en el caso de las especies forestales, el proceso consiste, primero, en jerarquizar a las cuatro especies agrícolas según su importancia económica. De acuerdo con este enfoque el orden jerárquico sería: 1) papa, 2) avena, 3) maíz y 4) haba.

Considerando esto, el proceso de determinación de zonas más adecuadas fue el mismo que en el caso anterior.

Así, se comparó cada especie con el resto y se asignaron las áreas donde dicha especie es dominante por encima de las demás.

Este análisis de dominancia se basó en un orden de jerarquía de acuerdo con la importancia relativa de cada especie agrícola basada en su rentabilidad económica.

De esta manera fue posible elaborar un mapa que señala las zonas agrícolas más adecuadas para cada género en las áreas que actualmente no tienen una cobertura forestal.

Así, se elaboró un mapa que indica qué zonas son más aptas para cada especie, generando de esta manera un mapa de distribución agrícola sugerida.

\section{Nivel de adecuación por especie en el PNNT}

La aplicación de la metodología antes descrita (figura 2) permitió obtener los mapas del nivel de adecuación del territorio para seis especies forestales y cuatro agrícolas.

\subsection{Aptitud para Pinus hartwegii}

Para el Pinus hartwegii el nivel de adecuación oscila entre 107 y 253. Como es posible apreciar en la figura $v$, la mayor parte del territorio del parque nacional presenta niveles altos de adecuación para esta especie. 
Figura $v$

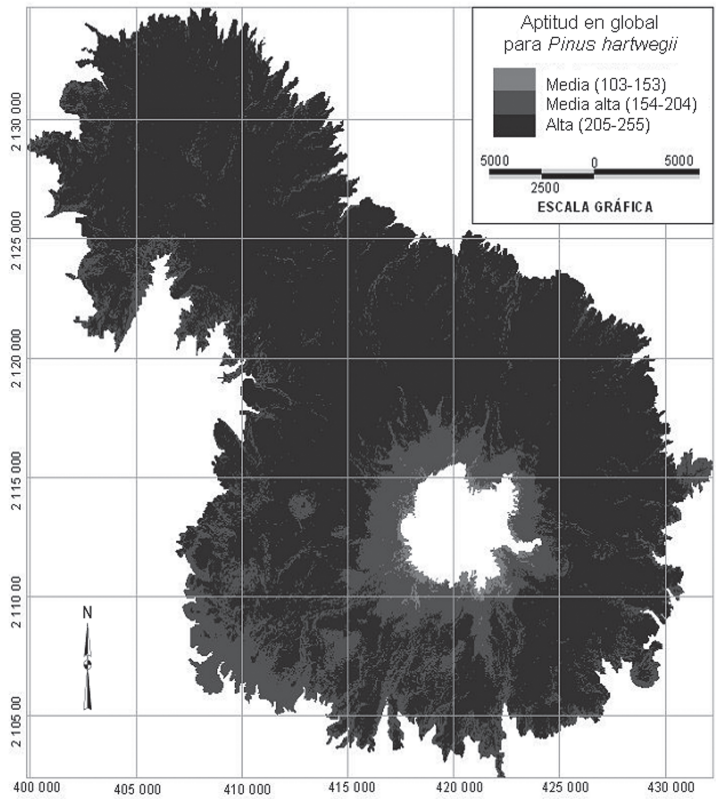

\subsection{Aptitud para Abies religiosa}

En el caso del Abies religiosa el nivel de adecuación oscila entre 130 y alcanza el máximo posible (255). Al igual que en el caso anterior, la mayor parte del parque tiene un nivel de adecuación alto. Sin embargo, evidentemente la altitud es una limitante importante (figura VI).

\subsection{Aptitud para Pinus montezumae}

El Pinus montezumae presenta un nivel de adecuación variable, los valores oscilan entre 91 y 255 . Teniendo en cuenta que esta especie tiene una clara limitación de establecimiento en altitud (hasta 3,150 msnmm), la mayor parte de las zonas en la que es factible su introducción tiene un nivel de adecuación de media a media alta (figura VII).

\subsection{Aptitud para Pinus ayacahuite}

Para Pinus ayacahuite los valores oscilan entre 80 hasta 184. Teniendo en cuenta que esta especie tiene una marcada limitación de establecimiento en altitud (hasta 3,250 $\mathrm{msnmm}$ ), en la mayor 


\section{Figura VI}

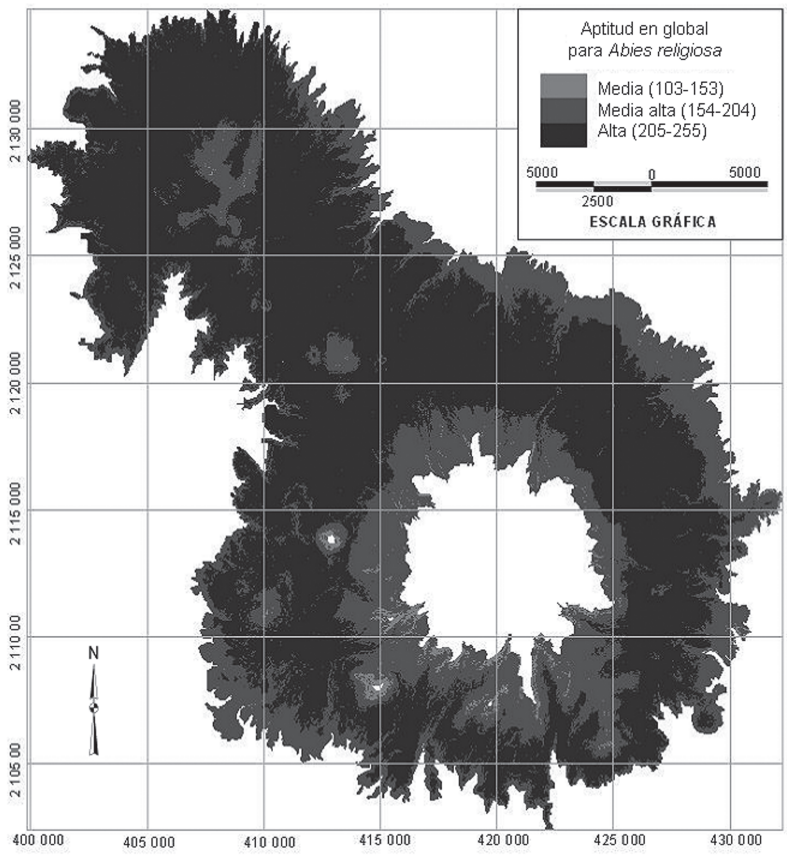

Figura VII

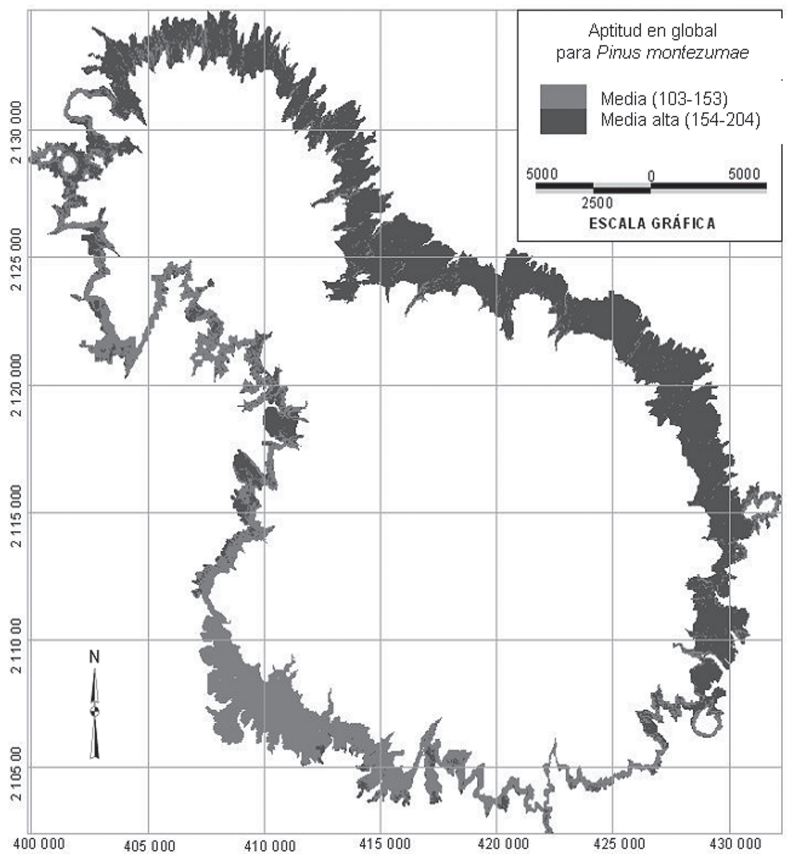


Figura VIII

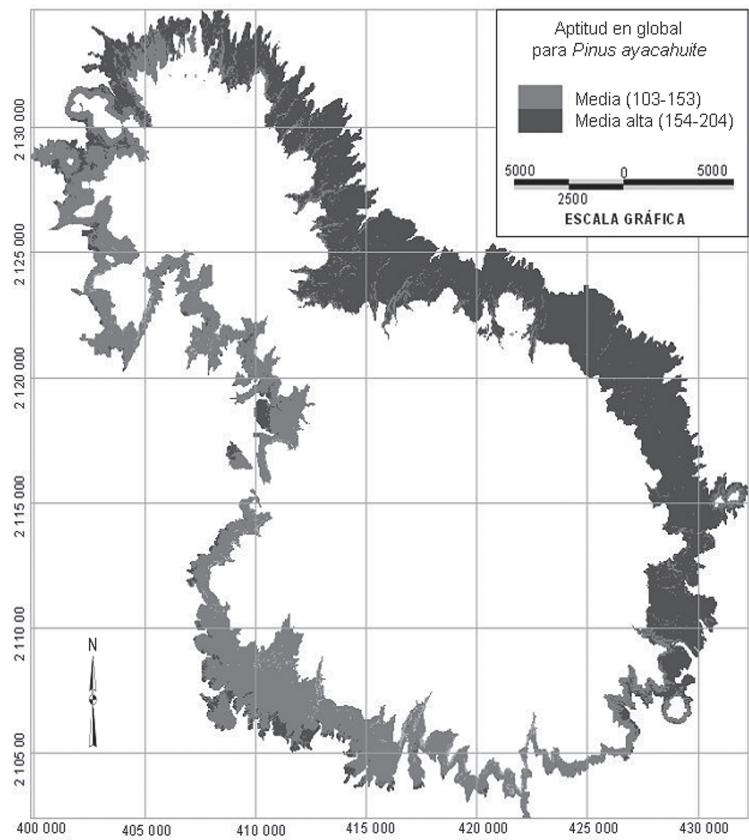

parte del territorio en el que es viable se tiene un nivel de adecuación de media a media alta (figura VIII).

\subsection{Aptitud para Alnus acuminata}

En lo que respecta al Alnus acuminata, los valores de adecuación oscilan desde 82 y alcanzan 255 . Como es posible ver, en la mayor parte del territorio de estudio se tiene un nivel de adecuación entre medio alto y alto, existiendo limitación en altitud conforme ésta aumenta (figura IX).

\subsection{Aptitud para Quercus laurina}

La adecuación para el Quercus laurina oscila entre 104 y 251. Se observa que en la mayor parte del territorio de estudio viable también se tiene un nivel de adecuación entre medio alto y alto, pero con una limitación en altitud más marcada (figura $\mathrm{x}$ ). 


\section{Figura IX}

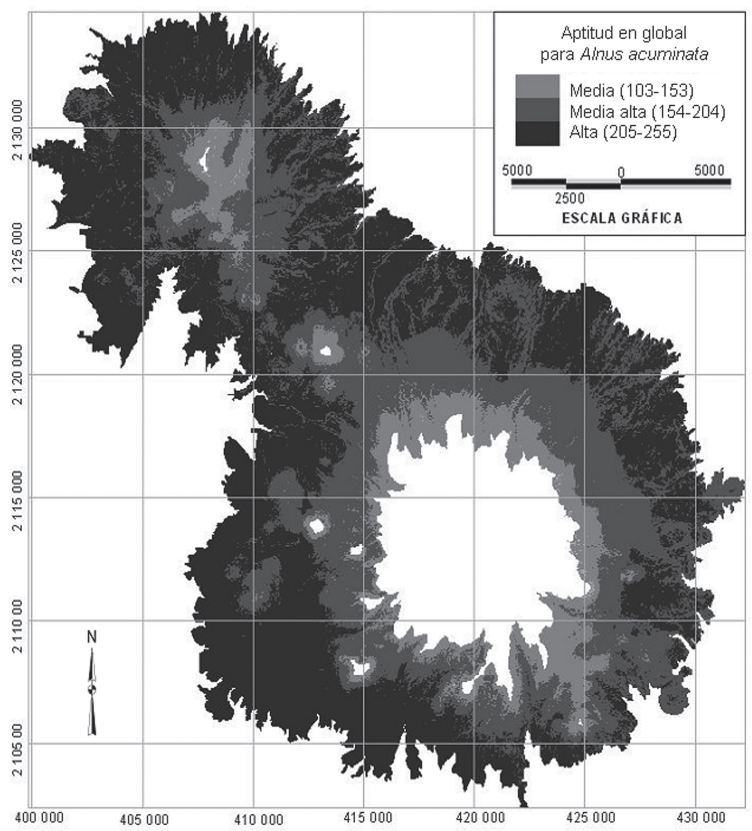

Figura $\mathrm{X}$

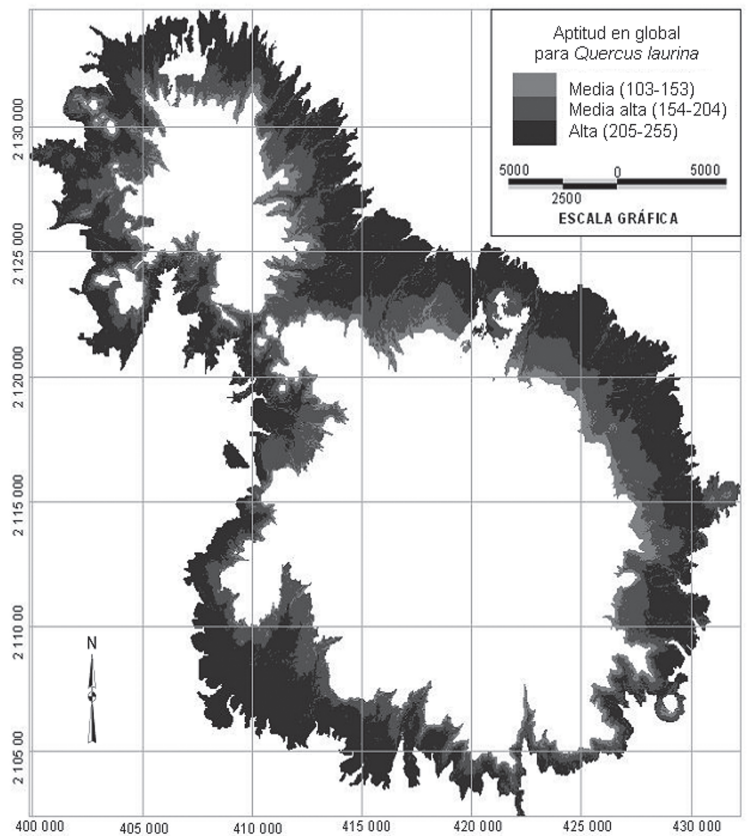




\subsection{Aptitud para Solanum tuberosum}

Para Solanum tuberosum el nivel de adecuación es mucho menor que lo reportado para las especies forestales. Los valores oscilan entre 31 y 163 . Se observa que en la mayor parte del territorio de estudio viable se tiene un nivel de adecuación entre medio y medio bajo, pero con una limitación en altitud marcada (figura XI).

\section{Figura XI}

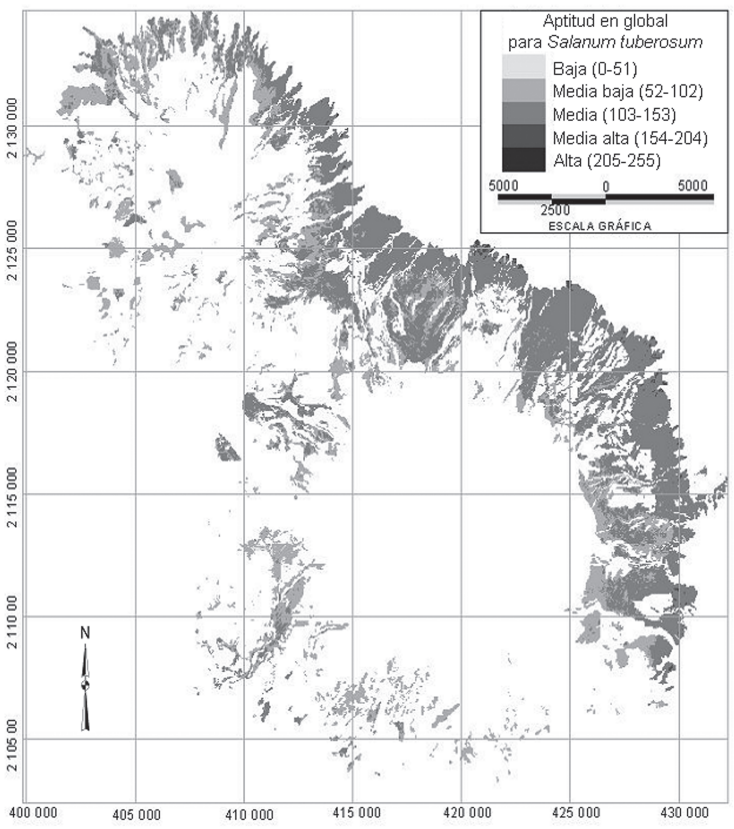

\subsection{Aptitud para Avena sativa}

La adecuación para Avena sativa también es, en términos generales, mucho menor que lo reportado para las especies forestales. Los valores oscilan entre 54 y 162, por lo que en cuanto a la adecuación global tiene una aptitud tendiente a media (figura XII).

\subsection{Aptitud para Zea mays}

Para Zea mays el nivel de adecuación también es, en términos generales, mucho menor que lo reportado para las especies forestales. Los valores oscilan entre 22 y 141, por lo que tiene una adecuación que tiende a media baja (figura XIII). 


\section{Figura XII}

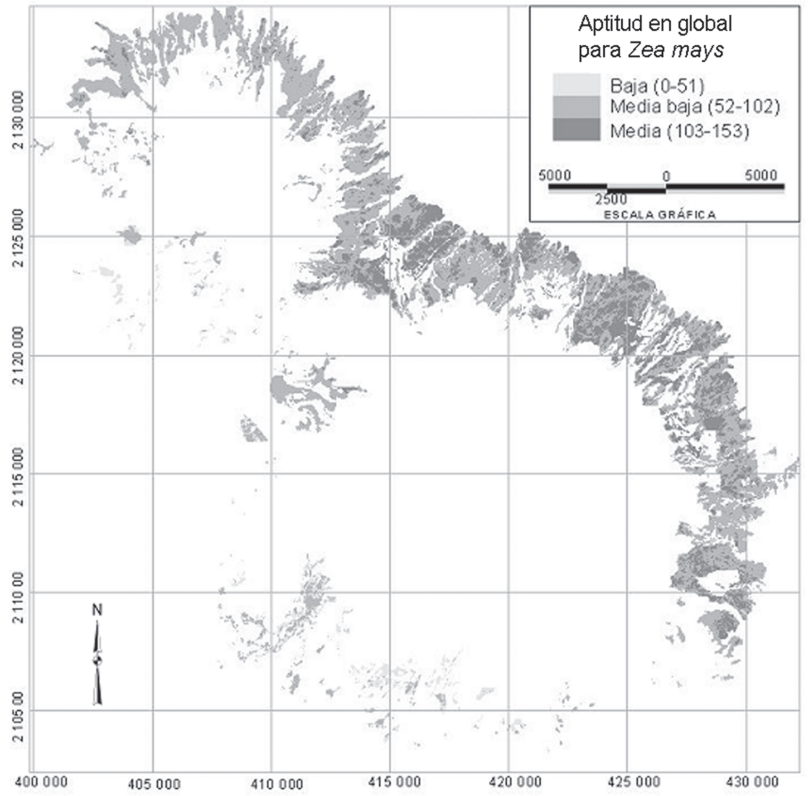

Figura XIII

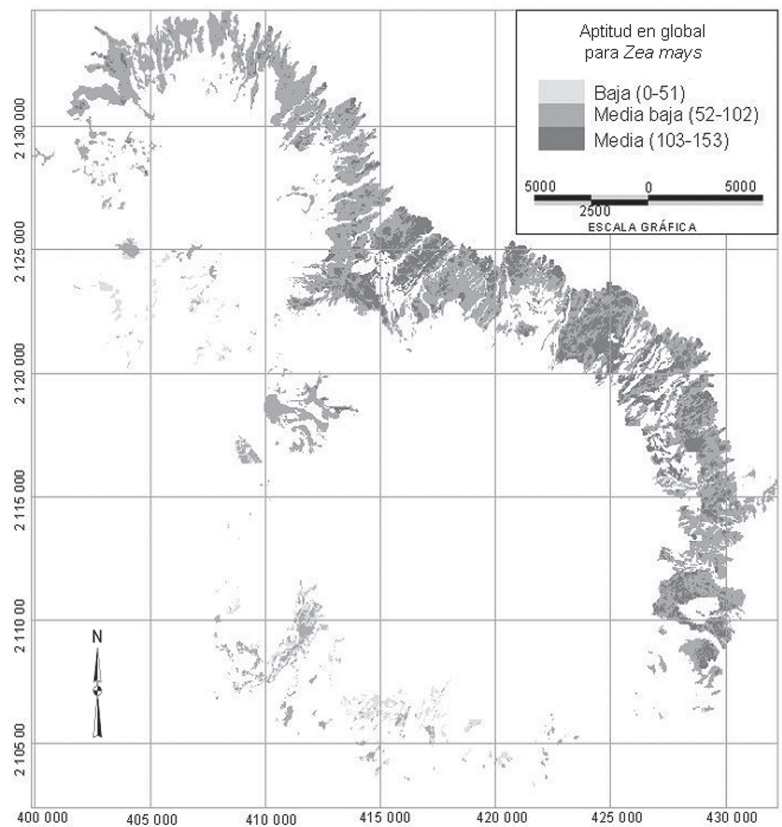




\subsection{Aptitud para Vicia faba}

En general, para Vicia faba el nivel de adecuación es mucho mayor que lo reportado para las otras tres especies agrícolas analizadas. Los valores oscilan entre 71 y 218 , con tendencia de media a media alta, por lo que su establecimiento dentro del PNNT parecería factible (figura XIV).

\section{Determinación de la distribución más adecuada de especies forestales}

Con la evidencia gráfica de que la vocación del área de estudio es eminentemente forestal, y considerando que en el análisis de dominancia las especies agrícolas tienden a desaparecer, la tarea siguiente fue determinar en qué áreas del parque nacional tiene una mejor aptitud territorial cada una de las cuatro principales especies forestales (Pinus harwegii, Abies religiosa, Alnus acuminata y Quercus laurina). Esto permitió, por añadidura, obtener como resultado un mapa de distribución forestal ideal según sus características climáticas, fisiográficas y edáficas. Con el procedimiento explicado antes, derivado de la comparación de niveles de adecuación, se obtuvo el mapa de distribución forestal ideal (figura Xv).

Como se puede apreciar, la configuración que debería tener el área de estudio sería de Pinus hartwegii en mayor proporción, cubriendo 30,343.92 hectáreas, Abies religiosa hacia la parte noroeste, con 8,697.08, Alnus acuminata hacia el noroeste, con 9,347.57 y finalmente Quercus laurina hacia el este del parque nacional, cubriendo 3,355.38 hectáreas.

\section{Distribución agrícola sugerida}

La obtención de la distribución óptima que deberían tener las principales especies forestales constituye una guía importante para desarrollar programas de reforestación en aquellas áreas que han perdido su ocupación original. Tal es el caso de las extensas zonas agrícolas que se encuentran en terrenos de vocación eminentemente forestal. La conversión de las zonas agrícolas en forestales, sin embargo, implica hacer frente a un problema socioeconómico importante. La agricultura en la región es fuente de subsistencia de ejidos y comunidades locales y, en este contexto, la reforestación de todo el parque nacional resulta una tarea poco realista. 


\section{Figura XIV}

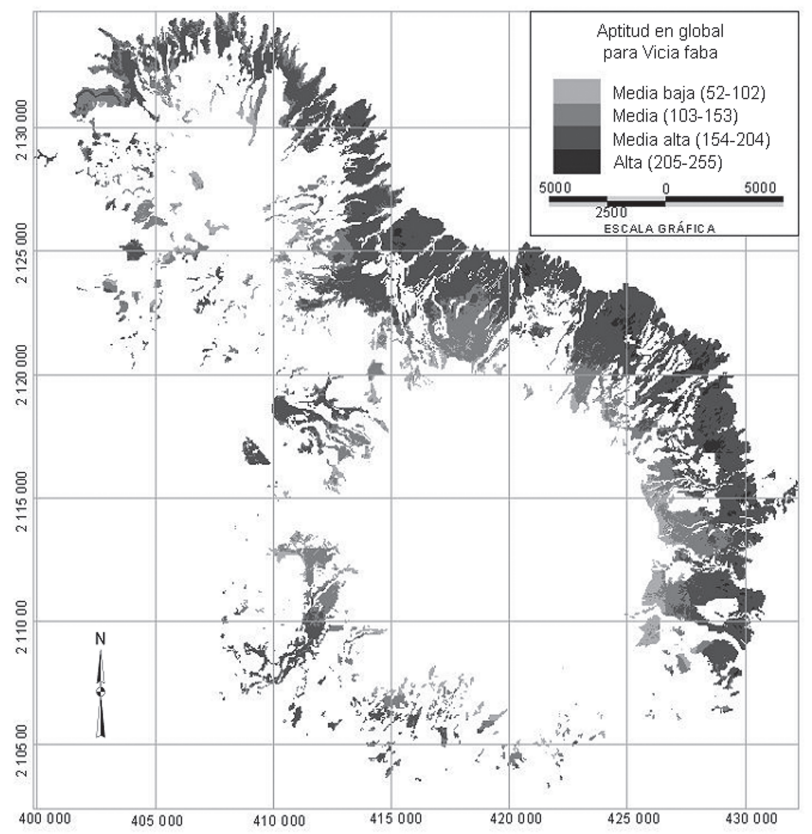

Figura XV

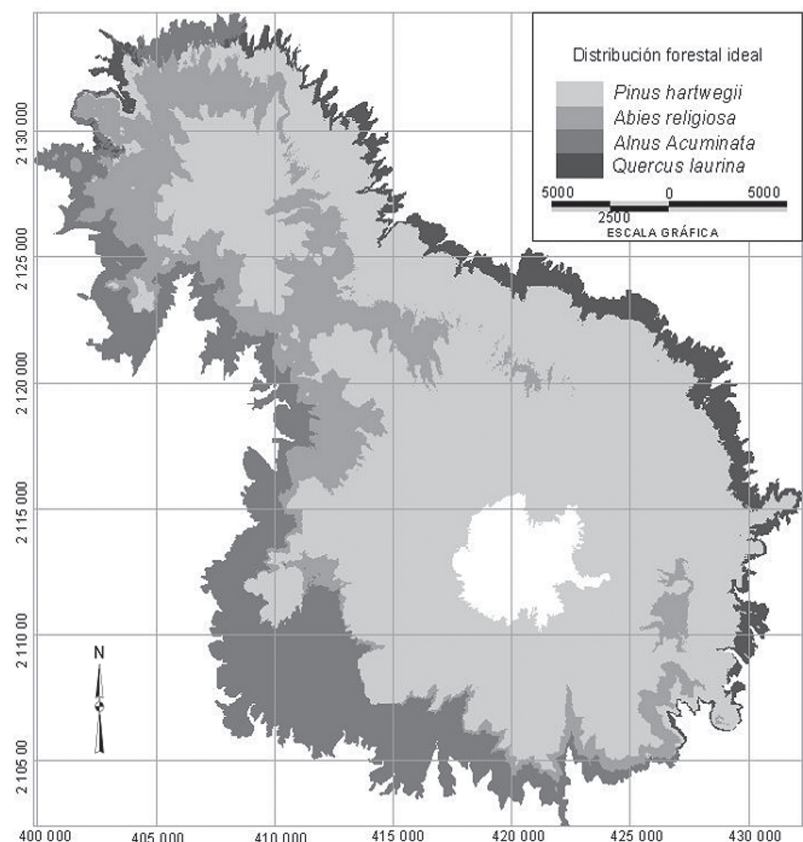


Por otra parte, los recorridos de campo demostraron que la producción agrícola en la región no necesariamente corresponde con el nivel de adecuación del territorio para dicha actividad. Por ejemplo, buena parte de las zonas agrícolas en el área natural protegida se destinan a la producción de maíz, sobre todo en zonas de gran altitud, en suelos frágiles y fuertes pendientes. En estas condiciones los rendimientos tienden a ser muy bajos y existen serios problemas de deterioro del suelo.

Ante esta situación se planteó la posibilidad de obtener el mapa de distribución agrícola óptima para los cuatro cultivos que tradicionalmente se practican en el Parque Nacional Nevado de Toluca: papa (Solanum tuberosum), avena (Avena sativa), maíz (Zea mays) y haba (Vicia faba). El análisis, por tanto, se centró en las áreas agrícolas y se basó en la comparación de niveles de adecuación para cada una de las especies. De esta manera se obtuvo el mapa de distribución agrícola en función de los mayores niveles de adecuación de dichas zonas (figura XVI).

Figura XVI

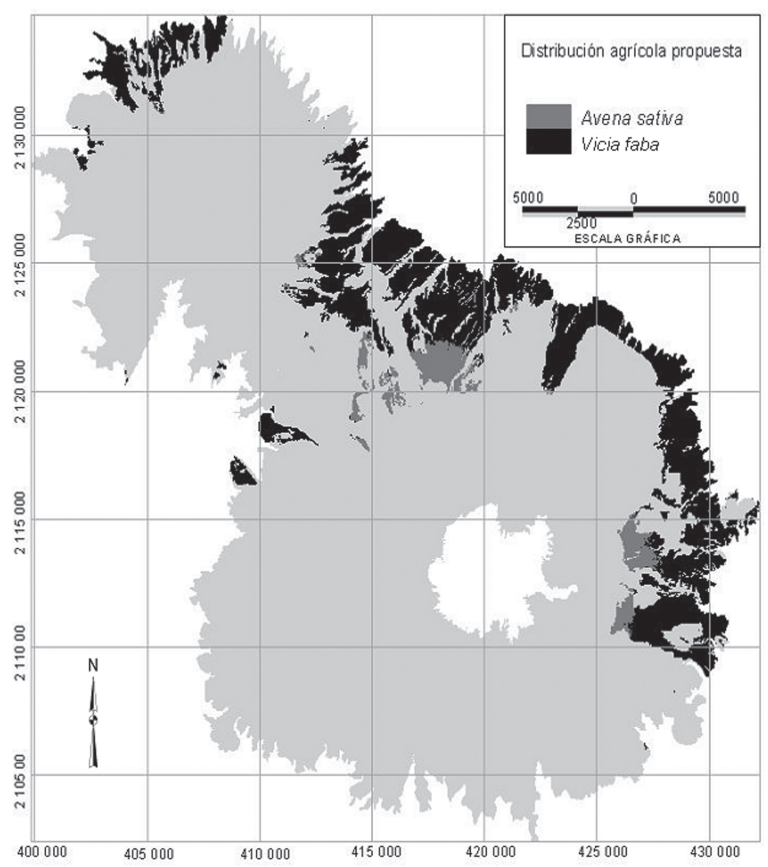


Como se puede observar en este mapa, dadas sus condiciones de suelo, clima y orografía, la región muestra niveles de adecuación más elevados para la producción de avena en las partes altas y haba en las partes bajas. Este resultado se atribuye al muy bajo nivel de aptitud del terreno para el cultivo de maíz y papa.

\section{Conclusiones}

Esta investigación tuvo como objetivo fundamental determinar el nivel de adecuación del Parque Nacional Nevado de Toluca para reintroducir especies forestales nativas y los principales cultivos en la región. Ello implicó definir criterios de análisis relativos al suelo, el clima y la orografía y realizar el análisis multicriterio mediante la aplicación de un sistema de información geográfica. La aplicación de esta herramienta permitió obtener la capacidad de acogida o nivel de adecuación territorial, como estrategia para plantear propuestas de ordenamiento territorial de los recursos forestales y agrícolas.

Evidentemente la metodología propuesta es perfectible y se puede complementar incorporando nuevas variables o criterios de análisis del nivel de adecuación del territorio. Ello implicaría la recopilación o generación de nueva información geográfica. Tal es el caso de información más detallada. Por ejemplo, es necesario contar con mayores datos sobre características fenológicas de las especies, fertilidad del suelo o variables meteorológicas como la evapotranspiración. Esto, sin embargo, queda más allá de los alcances de la investigación llevada a cabo.

Por supuesto uno de los aspectos fundamentales en todo análisis del nivel de adecuación del territorio radica en la disponibilidad de información cartográfica. En este sentido, la utilización de sistemas automatizados implicó un procesamiento de datos, digitalización de información analógica y la generación en formato digital de nueva información. Así, uno de los problemas principales para este trabajo fue la falta de cartografía disponible, suficientemente detallada y actualizada.

Por otra parte, en lo que respecta al procedimiento, el problema central fue la enorme complejidad resultante de evaluar el nivel de adecuación del territorio para 10 especies. Por ello, se trató de un largo proceso de análisis con la consiguiente generación de una gran cantidad de mapas intermedios. En este sentido, fue preciso desarrollar estrategias que permitieran mantener el control sobre todo el proceso. 
En lo tocante a los resultados del análisis, es pertinente señalar que casi toda la región muestra el mayor nivel de adecuación para el desarrollo de bosques de Pinus hartwegii. Esto resulta de especial relevancia dado que, como demuestran otros estudios, este tipo de bosque es el que se encuentra sometido a los más intensos procesos de deforestación y deterioro (Franco et al., 2006b). Abies religiosa fue la segunda especie forestal en cuanto a nivel de adecuación del territorio, sin embargo, por sus características fenológicas, presenta ciertas restricciones en lo que respecta a la altitud. Finalmente, como resultado del análisis, las condiciones regionales no parecieran favorecer el desarrollo de las otras dos especies de coníferas (Pinus montezumae y Pinus ayacabuite).

Para el caso de Alnus acuminata, algunas regiones bajas del suroeste del parque nacional muestran elevados niveles de adecuación, lo cual lo convierte en la tercera especie forestal en términos de su viabilidad. El Quercus laurina, por su parte, presenta mayores restricciones de altitud y sólo es viable en pequeñas porciones cercanas a la cota 3,000 msnm.

En cuanto a las especies agrícolas, su aptitud territorial en el Parque Nacional Nevado de Toluca es baja y nunca por encima de la vocación forestal. Destaca, sin embargo, el nivel de adecuación territorial del haba (Vicia faba), que en algunas ocasiones puede superar el nivel de adecuación para algunas especies de coníferas como Pinus montezumae y Pinus ayacahuite.

Finalmente es preciso señalar que, desde el punto de vista del nivel de adecuación del territorio para el desarrollo de especies agrícolas, se hace imprescindible la búsqueda de nuevas alternativas para el uso racional del territorio. Es decir, el manejo de cultivo como haba y avena debería considerar otras opciones de producción agrícola que tiendan a la recuperación de los recursos naturales. Por ello se debe continuar investigando acerca del ordenamiento de los sistemas agrícolas, lo cual rebasa los objetivos del presente estudio.

\section{Bibliografía}

Aragón, Luis Humberto (2003), Factibilidades agrícolas y forestales en la República Mexicana, Trillas, México.

Barba-Romero, Sergio y Jean Charles Pomerol (1997), Decisiones multicriterio. Fundamentos teóricos y utilización práctica, Universidad de Alcalá, Alcalá de Henares. 
Barredo, José Ignacio (1996), Sistemas de información geográfica y evaluación multicriterio en la ordenación del territorio, RA-MA, Madrid.

Conafor (Comisión Nacional Forestal) (2006), Fichas técnicas para la reforestación, Comisión Nacional Forestal, México, http://www.conafor.gob.mx/.

Eastman, J. Ronald, Peter Kyem y James Toledano (1993), “A procedure for Multi-Objective Decision Making in GIS Under Conditions of Conflicting objectives", European Conference of Geographical Information Systems, Munich, pp. 438-447.

Eastman, J. Ronald (1995), Idrisi for Windows, user's guide version 1.0, Clark University, Worcester, Mass.

Franco-Maass, Sergio (2003), Sistema de información geográfica para la localización de residuos peligrosos, UAEM, Toluca.

Franco-Maass, Sergio y Héctor Hugo Regil (2005), "Evaluación edafológico-climática para la introducción de frutales perennes y pastos retenedores de suelo en el Parque Nacional Nevado de Toluca”, Informe técnico del proyecto, Icamex, México.

Franco-Maass, Sergio, Héctor Hugo Regil, Carlos González y Gabino Nava (2006a), "Cambio de uso del suelo y vegetación en el Parque Nacional Nevado de Toluca, México, en el periodo 1972-2000”, Investigaciones Geográficas, Boletín del Instituto de Geografía, 61, Universidad Nacional Autónoma de México, México, pp. 38-57.

Franco-Maass, Sergio, Héctor Hugo Regil y José Antonio Benjamín Ordóñez (2006b), "Dinámica de perturbación-recuperación de las zonas forestales en el Parque Nacional Nevado de Toluca”, Madera y Bosques, 12 (1), Instituto de Ecología, A.C., México, pp. 17-28.

García, Enriqueta (1989), Apuntes de climatología, Universidad Nacional Autónoma de México, México. 
GEM (Gobierno del Estado de México) (1999), Programa de manejo del Parque Nacional Nevado de Toluca, Gobierno del Estado de México, México.

Gómez, David (1992), Evaluación de impacto ambiental, Agrícola Española, Madrid.

IMTA (Instituto Mexicano de Tecnología del Agua) (1996), Extractor rápido de información climatológica 2 (ERIC 2), Instituto Mexicano de Tecnología del Agua, México.

Luque, Ana María (2003), “La evaluación del medio para la práctica de actividades turístico-deportivas en la naturaleza", Cuaderno de Turismo, 12, Universidad de Málaga, Málaga, pp. 131-149.

Malczewski, Jacek (1999), GIS and multicriteria decision analysis, John Wiley \& Sons Inc., New Jersey.

Sánchez, Ángel y Alfredo Monte (1992), “Aplicación de un sistema de información geográfica en un estudio de acogida del territorio", Investigaciones geográficas, 10, unAM, México, pp. 215-225.

Santé, Inés y Rafael Crecente (2005), “Evaluación de métodos para la obtención de mapas continuos de aptitud para usos agroforestales", Geo Focus, 5, Grupo de Tecnologías de la Información Geográfica, Madrid, pp. 40-68.

Voogd, Henk (1983), Multicriteria Evaluation of Urban ad Regional Planning, Pion, Londres.

Recibido: 6 de marzo de 2008. Reenviado: 27 de febrero de 2009.

Aceptado: 2 de marzo de 2009.

Héctor Hugo Regil-García. Es maestro en ciencias agropecuarias y recursos naturales y licenciado en geografía y ordenación del territorio por la Universidad Autónoma del Estado de México (UAEM). Actualmente es becario de investigación en el Instituto de Ciencias Agropecuarias y Rurales de la UAEM. Sus líneas de 
investigación son: manejo y conservación de recursos naturales, cambio de uso de suelo y aplicaciones en sistemas de información geográfica. Entre sus publicaciones destacan: "Dinámica de perturbación-recuperación de las zonas forestales en el Parque Nacional Nevado de Toluca", Madera y Bosques, 12, Instituto de Ecología, A.C., México, pp. 17-28 (2006); "Cambio de uso de suelo y vegetación en el Parque Nacional Nevado de Toluca en el periodo 1972-2000", Investigaciones Geográficas, 61, Universidad Nacional Autónoma de México, México, pp. 38-57 (2006); "La evaluación multicriterio de los recursos turísticos en el Parque Nacional Nevado de Toluca”, Estudios y Perspectivas en Turismo, 18, Centro de Investigaciones y Estudios Turísticos. Buenos Aires, pp. 208-226 (2009).

Sergio Franco-Maass. Es maestro en Topographic Sciences por la Universidad de Glasgow y licenciado en diseño de asentamientos humanos por la Universidad Autónoma Metropolitana. Actualmente es investigador nivel I en el Sistema Nacional de Investigadores (SNI) y es secretario de investigación y estudios avanzados de la Universidad Autónoma del Estado de México. Sus líneas de investigación actuales son: manejo y conservación de recursos naturales en áreas naturales protegidas, aplicación de sistemas de información geográfica para la evaluación de recursos naturales y captura de carbono. Entre sus publicaciones destacan: "La evaluación multicriterio de los recursos turísticos en el Parque Nacional Nevado de Toluca", Estudios y Perspectivas en Turismo, 18, Centro de Investigaciones y Estudios Turísticos, Buenos Aires, pp. 208-226 (2009). "Payments for environmental services: an alternative for sustainable rural development? The case of a natural park in the central highlands of Mexico", Mountain Research and Development, 28 (1), Universidad de Berna, (2008), pp. 23-25 (2008); "Dinámica de perturbación-recuperación de las zonas forestales en el Parque Nacional Nevado de Toluca", Madera y Bosques, 12, Instituto de Ecología, A.C., México, pp. 17-28 (2006); entre otros. Ha colaborado en más de 10 capítulos de libros, y entre los publicados individualmente destacan: Principios básicos de cartografía y cartografía automatizada (2004) y Sistema de información geográfica para la localización de residuos peligrosos (2003). 